\title{
Shear Viscosity of claylike colloids in computer simulations and experiments
}

\section{Citation for published version (APA):}

Hecht, M., Harting, J. D. R., Bier, M., Reinshagen, J., \& Herrmann, H. J. (2006). Shear Viscosity of claylike colloids in computer simulations and experiments. Physical Review E - Statistical, Nonlinear, and Soft Matter Physics, 74(2), 021403-1/12. [021403]. https://doi.org/10.1103/PhysRevE.74.021403

DOI:

10.1103/PhysRevE.74.021403

Document status and date:

Published: 01/01/2006

\section{Document Version:}

Publisher's PDF, also known as Version of Record (includes final page, issue and volume numbers)

\section{Please check the document version of this publication:}

- A submitted manuscript is the version of the article upon submission and before peer-review. There can be important differences between the submitted version and the official published version of record. People interested in the research are advised to contact the author for the final version of the publication, or visit the $\mathrm{DOI}$ to the publisher's website.

- The final author version and the galley proof are versions of the publication after peer review.

- The final published version features the final layout of the paper including the volume, issue and page numbers.

Link to publication

\section{General rights}

Copyright and moral rights for the publications made accessible in the public portal are retained by the authors and/or other copyright owners and it is a condition of accessing publications that users recognise and abide by the legal requirements associated with these rights.

- Users may download and print one copy of any publication from the public portal for the purpose of private study or research.

- You may not further distribute the material or use it for any profit-making activity or commercial gain

- You may freely distribute the URL identifying the publication in the public portal.

If the publication is distributed under the terms of Article 25fa of the Dutch Copyright Act, indicated by the "Taverne" license above, please follow below link for the End User Agreement:

www.tue.nl/taverne

Take down policy

If you believe that this document breaches copyright please contact us at:

openaccess@tue.nl

providing details and we will investigate your claim. 


\title{
Shear viscosity of claylike colloids in computer simulations and experiments
}

\author{
Martin Hecht, ${ }^{1}$ Jens Harting, ${ }^{1}$ Markus Bier, ${ }^{2}$ Jörg Reinshagen, ${ }^{3}$ and Hans J. Herrmann ${ }^{4,5}$ \\ ${ }^{1}$ Institute for Computational Physics, Pfaffenwaldring 27, 70569 Stuttgart, Germany \\ ${ }^{2}$ Max-Planck-Institut für Metallforschung, Heisenbergstraße 3, 70569 Stuttgart, Germany \\ and Institut für Theoretische und Angewandte Physik, Universität Stuttgart, Pfaffenwaldring 57, 70569 Stuttgart, Germany \\ ${ }^{3}$ Institute of Ceramics in Mechanical Engineering, University of Karlsruhe (TH), Karlsruhe 76131, Germany \\ ${ }^{4}$ Institut für Baustoffe, Schafmattstrasse 6, ETH Zürich, CH-8093 Zürich, Switzerland \\ ${ }^{5}$ Departamento de Física, Universidade Federal do Ceará Campus do Pici, 60451-970 Fortaleza CE, Brazil
}

(Received 18 January 2006; revised manuscript received 2 May 2006; published 4 August 2006)

\begin{abstract}
Dense suspensions of small strongly interacting particles are complex systems that are rarely understood on the microscopic level. We investigate properties of dense suspensions and sediments of small spherical $\mathrm{Al}_{2} \mathrm{O}_{3}$ particles in a shear cell by means of a combined molecular-dynamics and stochastic rotation dynamics simulation. We study structuring effects and the dependence of the suspension's viscosity on the shear rate and shear thinning for systems of varying salt concentration and $p \mathrm{H}$ value. To show the agreement of our results with experimental data, the relation between the bulk $p \mathrm{H}$ value and surface charge of spherical colloidal particles is modeled by Debye-Hückel theory in conjunction with a $2 \mathrm{pK}$ charge regulation model.
\end{abstract}

DOI: 10.1103/PhysRevE.74.021403 PACS number(s): 82.70.-y, 47.11.-j, 47.57.Qk, 77.84.Nh

\section{INTRODUCTION}

We simulate colloids of silt particles, for which in many cases the attractive Van der Waals forces are relevant. These colloids are sometimes called "peloids" (Greek: claylike). In contrast to clays consisting of thin platelets [1], our particles are in first approximation spherical particles. For real clays, the particle shape and their orientation is of relevance [2-6]. For silt particles, on the other hand, the description is less complex. However, due to the particle size of micrometers and below, the interplay of diffusion, electrostatic repulsion, van der Waals attraction, and hydrodynamics still renders the suspension a very complex system. Colloid science tries to investigate the properties of such suspensions, and there is a vast amount of literature on this subject [7-11]. Colloids in general have various applications ranging from food industry over paintings and cosmetic products to applications in photographic processes. Particles with well defined properties can be used to investigate general properties of soft condensed matter like gelation or crystallization on a larger length scale than on the atomic level. Especially attractive interactions (depletion forces as well as van der Waals attraction) have attracted attention in the recent years [12-16]. In soil mechanics, real samples, e.g., of sediments, can be less characterized and therefore it is more difficult to gain a microscopic picture from which general properties can be derived. Therefore, we have chosen a synthetic $\mathrm{Al}_{2} \mathrm{O}_{3}$ powder suspended in water as a model system for silt. The particle diameter is $0.37 \mu \mathrm{m}$.

$\mathrm{Al}_{2} \mathrm{O}_{3}$ is not only a cheap testing material for investigations related to soil mechanics, but it is also an important material for ceramics. In process engineering, one of the basic questions is how to obtain components of a predefined shape. Wet processing of suspensions, followed by a sinter process, is a common practice here [17]. Nevertheless, to optimize the production process and to improve the homogeneity and strength of the fabricated workpiece, one has to understand the complex rheological behavior of the suspen- sion and its relation to the microscopic structure. This knowledge in turn can be applied to soil mechanics. Shear thinning as observed in our simulations and experiments is an important mechanism for the dynamics of landslides, making them more dangerous.

In this paper, we present our simulation results of sheared suspensions of $\mathrm{Al}_{2} \mathrm{O}_{3}$ particles. The overall behavior is strongly determined by the effective interaction potential between the particles in the suspension. The potentials can be related to experimental conditions within Debye-Hückel theory, and thus we can compare our simulation results to experimental data. In contrast to our approach of a direct comparison to experimental data, in the literature simulation results are often compared to analytical calculations.

Many different simulation methods have been developed and applied to colloidal suspensions: Stokesian dynamics (SD) [18-20], accelerated Stokesian dynamics (ASD) [21,22], pair drag simulations [23], Brownian dynamics (BD) [24,25], Lattice-Boltzmann method (LB) [26-29], and Stochastic rotation dynamics (SRD) [30-32]. Due to the complex nature of the problem, all simulation methods have to simplify in some point. Either Brownian motion is neglected or hydrodynamic interactions are included on a very simplified level. In many cases, simulations are done without a quantitative comparison to experiments. In the present paper, we combine molecular dynamics (MD) to simulate the colloidal particles, SRD for the description of the fluid, and a charge regulation model that provides us with realistic parameters for the Derjaguin-Landau-Vervey-Overbeek (DLVO) potentials $[33,34]$ in the MD simulation. We include long-range hydrodynamic interactions on a coarse-grained level in the SRD part, and we only include DLVO pair potentials in the MD part. No electrostatic many-body interactions or electrodynamic interactions are considered, and modifications of the pair potentials due to locally increased colloid concentrations are neglected, too. However, many numerical investigations are based on much simpler models than ours. In our opinion, our model covers the main properties quite well. 
Our paper is organized as follows. First we briefly describe our MD implementation, followed by a short sketch of the SRD simulation method and a description of how we have implemented our shear cell. The simulation method is described in detail in Ref. [30]. Then we describe the so-called $2 \mathrm{pK}$ charge regulation model, which relates our simulation parameters with the $p \mathrm{H}$ value and the ionic strength $I$ adjusted in the experiment. A short description of the simulation setup and of the experiments carried out follows. After that, we present our simulation results and compare them to the experimental data. Finally, a summary is given.

\section{MOLECULAR DYNAMICS}

We study colloidal particles, composing the solid fraction, suspended in a fluid solvent. The colloidal particles are simulated with molecular dynamics (MD), whereas the solvent is modeled with stochastic rotation dynamics (SRD) as described in Sec. III.

In the MD part of our simulation, we include effective electrostatic interactions and van der Waals attraction, a lubrication force, and Hertzian contact forces. The electrostatic and van der Waals potential are usually referred to as DLVO potentials $[8-10,24,35,36]$, which capture the static properties of colloidal particles in aqueous suspensions. The first component is the screened Coulomb term

$$
\begin{aligned}
V_{\text {Coul }}= & \pi \varepsilon_{r} \varepsilon_{0}\left[\frac{2+\kappa d}{1+\kappa d} \frac{4 k_{B} T}{z e} \tanh \left(\frac{z e \zeta}{4 k_{B} T}\right)\right]^{2} \\
& \times \frac{d^{2}}{r} \exp (-\kappa[r-d]),
\end{aligned}
$$

where $d$ denotes the particle diameter and $r$ is the distance between the particle centers. $e$ is the elementary charge, $T$ is the temperature, $k_{B}$ is the Boltzmann constant, and $z$ is the valency of the ions of added salt. Within DLVO theory, one assumes linear screening, mainly by one species of ions with valency $\pm z$ (e.g., $z=+1$ for $\mathrm{NH}_{4}^{+}$). The first fraction in Eq. (1) is a correction to the original DLVO potential, which takes the surface curvature into account and is valid for spherical particles [37].

The effective surface potential $\zeta$ is the electrostatic potential at the border between the diffuse layer and the compact layer. It may therefore be identified with the $\zeta$ potential. It includes the effect of the bare charge of the colloidal particle itself, as well as the charge of the ions in the Stern layer, where the ions are bound permanently to the colloidal particle. In other words, DLVO theory uses a renormalized surface charge, which we determine by the model described in Sec. IV.

$\kappa$ is the inverse Debye length defined by $\kappa^{2}=8 \pi \ell_{B} I$, with the ionic strength $I$. The Bjerrum length $\ell_{B}:=\frac{\beta e^{2}}{4 \pi \varepsilon_{0} \varepsilon_{r}}$ measures the distance at which the electrostatic interaction of two elementary charges amounts to $\beta^{-1}=k_{B} T$. $\varepsilon_{0}$ is the permittivity of the vacuum and $\varepsilon_{r}$ is the relative dielectric constant of the solvent (we use 81 for water, i.e., $\ell_{B}=7 \AA$ for room temperature).
The Coulomb term of the DLVO potential competes with the attractive van der Waals term

$$
V_{\mathrm{VdW}}=-\frac{A_{H}}{12}\left[\frac{d^{2}}{r^{2}-d^{2}}+\frac{d^{2}}{r^{2}}+2 \ln \left(\frac{r^{2}-d^{2}}{r^{2}}\right)\right] .
$$

$A_{H}=4.76 \times 10^{-20} \mathrm{~J}$ is the Hamaker constant [25], which involves the polarizability of the particles. The singularity of $V_{\mathrm{VdW}}$ for touching particles is removed and the primary minimum is modeled by a parabola as described in Ref. [30].

Long-range hydrodynamic interactions are taken into account in the simulation for the fluid as described below. This can only reproduce interactions correctly down to a certain length scale. On shorter distances, a lubrication force has to be introduced explicitly in the MD simulation. The most dominant mode, the so-called squeezing mode, is an additional force

$$
\mathbf{F}_{\text {lub }}=-\left(\mathbf{v}_{\text {rel }}, \hat{\mathbf{r}}\right) \hat{\mathbf{r}} \frac{6 \pi \eta}{r-d}\left(\frac{R}{2}\right)^{2}
$$

between two particles with radius $R$ and relative velocity $\mathbf{v}_{\text {rel }}$. $\eta$ is the dynamic viscosity of the fluid. In contrast to the DLVO potentials, the lubrication force is a dissipative force. When two particles approach each other very closely, this force becomes very large. To ensure numerical stability of the simulation, one has to limit $\mathbf{F}_{\text {lub }}$. We choose a maximum force at a certain gap width $r_{\mathrm{sc}}$ and shift the force so that the maximum force cannot be exceeded: Instead of calculating $\mathbf{F}_{\text {lub }}(r)$, we take the value for $\mathbf{F}_{\text {lub }}\left(r+r_{\text {sc }}\right)$. In addition, since the force decays for large particle distances, we can introduce a large cutoff radius $r_{\text {lc }}$ for which we assume $\mathbf{F}_{\text {lub }}(r)$ $\equiv 0$ if $r-d>r_{\mathrm{lc}}$. As the intention of $\mathbf{F}_{\text {lub }}$ is to correct the finite resolution of the fluid simulation, $r_{\mathrm{sc}}$ and $r_{\mathrm{lc}}$ have to be adjusted in a way that the dynamic properties, i.e., the viscosity of a simulated particle suspension with weak DLVO interactions, fits the measurements. It turns out that $r_{\mathrm{sc}}=\frac{d}{40}$ and $r_{\mathrm{lc}}=\frac{3}{2} d$ work best. Our approach for $\mathbf{F}_{\text {lub }}$ is similar to the one often used in lattice Boltzmann simulations [26]. In contrast to Ladd [26], we have chosen to use two cutoff radii to be able to treat small and large gaps separately. There are different approaches, e.g., for Stokesian dynamics [18], where the force field is expanded to a multipole series and the far field part is subtracted afterwards.

Finally, we use a Hertz force described by the potential

$$
V_{\text {Hertz }}=K(d-r)^{5 / 2} \text { if } r<d,
$$

where $K$ is the constant that describes the elasticity of the particles in the simulation. The Hertz force avoids that the particles penetrate each other. It also contains a damping term in normal direction,

$$
\mathbf{F}_{\text {Damp }}=-\left(\mathbf{v}_{\text {rel }}, \hat{\mathbf{r}}\right) \hat{\mathbf{r}} \beta_{D} \sqrt{d-r},
$$

with a damping constant $\beta_{D}$.

Since in this work no stress perpendicular to the shear direction is applied, the tangential forces at the particle surface are not of essential importance. To verify this, we have increased the spacial resolution of the fluid simulation, included tangential forces on the particles, and allowed particle rotations. Even though the computational effort was consid- 
erably larger, and one could expect that more effects on the length scale below the particle diameter could be covered, one could observe only a change of some percent in the viscosity and in the velocity profile. Due to the DLVO potential and the lubrication force, the particles very rarely get into contact as long as no confining stress is applied. The only case in which particles really touch each other, would be if the $\zeta$ potential is close to zero at a certain $p \mathrm{H}$ value. This $p \mathrm{H}$ value is called "isoelectric point." It depends on the material of the suspended particles and on the solvent. For our system it is at $p \mathrm{H}=8.7$ [36]. In experiments close to the isoelectric point, a solid fraction immediately flocculates out and sediments. In the simulation, one ends up with only one big cluster in the simulation volume, which corresponds to a part of a floc seen in the experiment.

For this study, we do not apply tangential forces and thus, having only central forces, we could neglect rotation of the particles. This reduces the computational effort substantially.

\section{STOCHASTIC ROTATION DYNAMICS (SRD): SIMULATION OF THE FLUID}

The stochastic rotation dynamics method (SRD) was first introduced by Malevanets and Kapral [38,39]. The method is also known as "real-coded lattice gas" [31] or as "multiparticle-collision dynamics" (MPCD) and has been successfully applied to simulate many important systems such as complex fluids containing polymers [40,41], vesicles in flow [42], and dynamics of chemical reactions [43]. The method is a promising tool for a coarse-grained description of a fluctuating solvent, e.g., in Ref. [44] the results of simulations of a flow around a cylinder are presented, or in Ref. [32] sedimentation of a particle suspension is studied.

The method is based on so-called fluid particles with continuous positions and velocities. Each time step is composed of two simple steps: one streaming step and one interaction step. In the streaming step the positions of the fluid particles are updated as in the Euler integration scheme known from molecular-dynamics simulations,

$$
\mathbf{r}_{i}(t+\tau)=\mathbf{r}_{i}(t)+\tau \mathbf{v}_{i}(t),
$$

where $\mathbf{r}_{i}(t)$ denotes the position of the particle $i$ at time $t$, $\mathbf{v}_{i}(t)$ is its velocity at time $t$, and $\tau$ is the time step used for the SRD simulation. After updating the positions of all fluid particles, they interact collectively in an interaction step that is constructed to preserve momentum, energy, and particle number. The fluid particles are sorted into cubic cells of a regular lattice and only the particles within the same cell are involved in the interaction step. First, their mean velocity $\mathbf{u}_{j}\left(t^{\prime}\right)=\frac{1}{N_{j}\left(t^{\prime}\right)} \sum_{i=1}^{N_{j}\left(t^{\prime}\right)} \mathbf{v}_{i}(t)$ is calculated, where $\mathbf{u}_{j}\left(t^{\prime}\right)$ denotes the mean velocity of cell $j$ containing $N_{j}\left(t^{\prime}\right)$ fluid particles at time $t^{\prime}=t+\tau$. Then, the velocities of each fluid particle in cell $j$ are updated as

$$
\mathbf{v}_{i}(t+\tau)=\mathbf{u}_{j}\left(t^{\prime}\right)+\boldsymbol{\Omega}_{j}\left(t^{\prime}\right) \cdot\left[\mathbf{v}_{i}(t)-\mathbf{u}_{j}\left(t^{\prime}\right)\right] .
$$

$\boldsymbol{\Omega}_{j}\left(t^{\prime}\right)$ is a rotation matrix, which is independently chosen randomly for each time step and each cell. We use rotations about one of the coordinate axes by an angle $\pm \alpha$, with $\alpha$ fixed [45]. The coordinate axis as well as the sign of the rotation are chosen at random, resulting in six possible rotation matrices. The mean velocity $\mathbf{u}_{j}(t)$ in the cell $j$ can be seen as the streaming velocity of the fluid at the position of the cell $j$ at the time $t$, whereas the difference $\left[\mathbf{v}_{i}(t)-\mathbf{u}_{j}\left(t^{\prime}\right)\right]$ entering the interaction step can be interpreted as a contribution to the thermal fluctuations. Thus, to calculate the local temperature in the cell under consideration, one has to sum over the squares of this expression.

The method just described is able to reproduce hydrodynamics and thermal fluctuations. To couple the colloidal particles to the streaming field of the solvent, we use "Coupling II" of Ref. [30]: we modify the rotation step of the original SRD algorithm slightly. The colloidal particles are sorted into the SRD cells as well and their velocity enters into the calculation of the mean velocity $\mathbf{u}_{j}(t)$ in cell $j$. Since the mass of the fluid particles is much smaller (in our case it is 250 times smaller) than the mass of the colloidal particles, we have to use the mass of each particle-colloidal or fluid particle-as a weight factor when calculating the mean velocity,

$$
\begin{gathered}
\mathbf{u}_{j}\left(t^{\prime}\right)=\frac{1}{M_{j}\left(t^{\prime}\right)} \sum_{i=1}^{N_{j}\left(t^{\prime}\right)} \mathbf{v}_{i}(t) m_{i}, \\
\text { with } M_{j}\left(t^{\prime}\right)=\sum_{i=1}^{N_{j}\left(t^{\prime}\right)} m_{i},
\end{gathered}
$$

where we sum over all colloidal and fluid particles in the cell, so that $N_{j}\left(t^{\prime}\right)$ is the total number of both particles together. $m_{i}$ is the mass of the particle with index $i$ and therefore $M_{j}\left(t^{\prime}\right)$ gives the total mass contained in cell $j$ at the time $t^{\prime}=t+\tau$. The update rule for the particle velocities $\mathbf{v}_{i}(t)$ and positions $\mathbf{r}_{i}(t+\tau)$, which we apply, is summarized in Eqs. (6)-(9). This method to couple some embedded material to the SRD simulation is described for different applications in the literature $[46,47]$.

This coupling method does not enforce no-slip boundaries on the particle surface, as in the method suggested by Inoue et al. [31]. Moreover, as very recently discussed by Padding and Louis [48], purely radial interactions effectively introduce slip boundary conditions. Considering the drag coefficient, a prefactor changes and this could be corrected by assuming a different hydrodynamic radius. We have checked the influence of hydrodynamic interactions by removing the fluid completely and by varying the resolution of the SRD simulation. Also, two different coupling methods as described in Ref. [30] have been applied. Without fluid, the achieved shear rate as well as the viscosity differed strongly, whereas the difference between the two coupling methods was in the order of some percent only. Therefore, we need hydrodynamics to some extent, but we have chosen the coupling method with less computational effort. Very recently, Yamamoto et al. have shown that for colloidal gelation, hydrodynamic interactions are of minor importance [49] for 3D systems, but in contrast to our work, they focus on the static properties of a colloidal system quenched to zero temperature. 
In Ref. [30], we have described a simple method to introduce shear at the fluid boundary by adding a velocity offset to all fluid particles reflected at the shear plane. From a constant velocity offset $\Delta \mathbf{v}$ one can calculate the mean shear force

$$
\mathbf{F}_{\mathrm{S}}=\left\langle\sum_{i=1}^{L} m_{i} \frac{\Delta \mathbf{v}_{i}(t)}{\tau}\right\rangle,
$$

where $L$ denotes the average number of fluid particles crossing through the shear plane in one time step and $\langle\cdots\rangle$ stands for a time average. $L$ can be expressed by the mean free path and the number density of fluid particles. This would be a force driven shear, where one has only indirect control on the shear rate $\dot{\gamma}$ or the shear velocity $\mathbf{v}_{S}$, respectively. Therefore, we modify the mean velocity $\mathbf{u}_{j}\left(t^{\prime}\right)$ in the cells close to the shear plane by changing the velocity of each fluid particle as well as the velocity of the colloidal particles contained in that specific cell by the difference $\mathbf{v}_{S}-\mathbf{u}_{j}\left(t^{\prime}\right)$. By construction, the mean velocity in these cells is equal to the shear velocity $\mathbf{v}_{S}$ after that step. At the wall itself we implement full slip boundary conditions for the fluid and for the colloidal particles. The boundary in the direction of the shear profile (direction of the velocity gradient) is chosen to be nonperiodic. By doing so, we can also observe phenomena such as wall-slip, nonlinear velocity profiles, or density profiles in our shear cell (see Sec. VII C). In the case of a nonlinear velocity profile, the viscosity is not well defined. We extract the central region of the profile where it is in first approximation linear and estimate there an averaged viscosity. This is the ratio of the velocity gradient and the shear force, which can be calculated in analogy to Eq. (10) by carrying out the sum over all velocity changes made. The region where we estimate the velocity gradient is half the system size.

We have tested a number of boundary conditions and different ways to impose shear, but the method just described turned out to work best. No-slip boundaries at a top and bottom plane seemed to work for high volume fractions and unless the potentials get attractive. As soon as (only slight) cluster formation sets in, the particles concentrate in the center of the system and lose contact with the sheared walls. Shearing only the fluid and not the colloidal particles always works, but the resulting viscosity is much too small. In fact, what one measures is the flow of the fluid streaming around the particles like a flow through a porous medium. The next point is how to determine the shear force and the velocity gradient we need for the calculation of the shear viscosity. The force is always related to any velocity changes made in the system, and its calculation is straightforward in most cases.

The imposed velocity difference divided by the system size perpendicular to the shear plane would give an averaged gradient. For clustered systems, not even the shape of the shear viscosity against shear rate was comparable to the measurements. If the velocity gradient changes within the system (compare Fig. 4), we have to take care that we measure the viscosity in the bulk, i.e., that we take the velocity gradient there. At least if the particles are not too strongly clustered, the slope of the plateau in the center of the system can be taken as a "good" velocity gradient. We use this velocity gradient as achieved shear rate as mentioned above. With this scheme for strongly attractive forces, the obtained viscosity $\eta(\dot{\gamma})$ for the simulation stays in the vicinity of the measured curve, whereas for other methods we tried out, the points of the simulation usually ended up far off the measured curve.

Fully periodic boundary conditions for sheared systems, known as Lees-Edwards conditions, would be a good choice for stable suspensions. As soon as clusters are formed, the velocity profile becomes nonlinear, as discussed above. But, additionally the location of the cluster, i.e., the position of the plateau, is not fixed anymore to the center of the system, which makes it more difficult to extract the correct velocity gradient. In addition, the shear force would be determined from the velocity changes of the particles passing around the periodic boundaries. If the cluster by chance stays in the center of the system, again only the fluid would be sheared and only indirectly, transmitted by the fluid, the force would be exerted on the particles, as if with closed boundaries only the walls would move and no sheared regions close to the wall were implemented. Together with the periodic boundaries, this would lead to large fluctuations of the shear force, caused by the present position of the cluster. Furthermore, the boundary conditions would have to be consistent for the MD and for the SRD simulation. For the MD part, it is important that the position, where a particle reenters the system after passing around the periodic boundary, is shifted by $2 t \mathbf{v}_{S}$, with $t$ being the continuously increasing simulation time and $\mathbf{v}_{S}$ the shear velocity. Additionally, this shift has to be wrapped around the periodic boundaries in shear direction. If we do the same with the fluid particles, the shift could be any value, not necessarily an integer multiple of the fluid box size. What we want to point out is that without any further restrictions, the grid in the SRD rotation step would no longer be regular in this plane, which in addition is the plane where one measures the shear force. To overcome this problem, one can restrict the shear rate to values determined by the SRD grid size and the SRD time step, but the other difficulties mentioned before remain.

\section{THE CHARGE REGULATION MODEL}

To determine the effective surface potential that enters the DLVO potential, we use the model described in the following. In reality, the surface charge is achieved by adsorption and desorption of charge determining ions leading to an electrostatic potential difference between surface and bulk, which in turn influences ion adsorption. A full description of this regulation of surface charges requires two parts: the first part describes the relation between surface charge density and surface potential due to the electrolytic environment, whereas the second part quantifies the ion adsorption depending on the surface concentration of charge-determining ions.

Concerning the first part, a relation between the surface charge density $\sigma$ and the surface potential $\zeta$ of a charged spherical colloidal particle of radius $R$ immersed in an electrolytic environment of relative dielectric constant $\varepsilon_{r}$ and ionic strength $I$ is given within Debye-Hückel theory [50,51] by 


$$
\zeta=\frac{R \sigma}{\varepsilon_{0} \varepsilon_{r}(1+\kappa R)} .
$$

As mentioned above, we consider the Stern layer as a part of the surface charge. Thus we can identify the effective surface potential in DLVO theory with the $\zeta$ potential, and we can thus skip a discussion of bare charge versus effective charge [52-54].

In the second part of our model, the adsorption of chargedetermining ions on the surface of the colloidal particle is described by assuming that the only mechanism of adsorption is that of protons $\left(\mathrm{H}^{+}\right)$on surface sites $(-\mathrm{S})$. It turned out that this assumption leads to reasonable results for surfaces made of $\mathrm{Al}_{2} \mathrm{O}_{3}$. Adsorption is described by the two chemical reactions [55]

$$
\begin{gathered}
-\mathrm{S}^{-}+\mathrm{H}^{+} \rightleftharpoons-\mathrm{SH}, \\
-\mathrm{SH}+\mathrm{H}^{+} \rightleftharpoons-\mathrm{SH}_{2}^{+},
\end{gathered}
$$

with the two reaction constants

$$
\begin{aligned}
& K_{1}:=\frac{\left[-\mathrm{S}^{-}\right]\left[\mathrm{H}^{+}\right] \exp (-\beta e \zeta)}{[-\mathrm{SH}]}, \\
& K_{2}:=\frac{[-\mathrm{SH}]\left[\mathrm{H}^{+}\right] \exp (-\beta e \zeta)}{\left[-\mathrm{SH}_{2}^{+}\right]} .
\end{aligned}
$$

In terms of the surface site concentrations, the total number of surface sites per area and the surface charge density are given by $N_{S}=\left[-\mathrm{S}^{-}\right]+[-\mathrm{SH}]+\left[-\mathrm{SH}_{2}^{+}\right]$and $\sigma=-e\left[-\mathrm{S}^{-}\right]$ $+e\left[-\mathrm{SH}_{2}^{+}\right]$, respectively. Defining $p K_{1}:=-\log _{10}\left(K_{1}\right)$ and $p K_{2}:=-\log _{10}\left(K_{2}\right)$ yields the point of zero charge $p \mathrm{H}_{z}$, i.e., the $p \mathrm{H}$ value of vanishing surface charge, as $p \mathrm{H}_{z}=\frac{1}{2}\left(p K_{1}\right.$ $\left.+p K_{2}\right)$. The surface site density $N_{S}$ and the difference $\Delta p K:=p K_{1}-p K_{2}$ are treated as adjustable parameters.

The above equations lead to the relation

$$
\frac{\sigma}{e N_{S}}=\frac{\delta \sinh \left(\psi_{N}-\beta e \zeta\right)}{1+\delta \cosh \left(\psi_{N}-\beta e \zeta\right)}
$$

with the Nernst potential $\psi_{N}:=\ln (10)\left(p \mathrm{H}_{z}-p \mathrm{H}\right)$ and $\delta:=2$ $\times 10^{-\Delta p K / 2}$.

Equations (11) and (16) can be solved self-consistently for $\zeta$ as a function of $p \mathrm{H}$. For our system of $\mathrm{Al}_{2} \mathrm{O}_{3}$ particles, we find $\Delta p K=4.2$ and $N_{S}=0.22 / \mathrm{nm}^{2}$. With these values the measured $\zeta$ potential of $52 \mathrm{mV}$ at $p \mathrm{H}=6, I=0.01 \mathrm{~mol} / 1$ and up to $120 \mathrm{mV}$ at $p \mathrm{H}=4, I=0.01 \mathrm{~mol} / \mathrm{l}$ can be reproduced best. For the experimental determination of the $\zeta$ potential, electrophoretic (Delsa 440SX, Beckman-Coulter GmbH, Germany) and electrokinetic measurements (AcustoSixer IIs, Colloidal Dynamics Ind., USA) were performed. To calculate the $\zeta$ potential, Henry's theory [8] was used. For details, see Ref. [56]. We have to admit that the relation between the directly measured quantities, e.g., electrophoretic mobility, and the $\zeta$ potential is a subject of current research [57-60].

\section{SIMULATION SETUP}

In our simulation, we try to model the experimental system as accurately as possible. We start with spherical par- ticles of diameter $d=0.37 \mu \mathrm{m}$, the mean diameter of the particles used in the experiment. The simulation box is $48 d$ $=17.76 \mu \mathrm{m}$ long in the $x$ direction, $24 d=8.88 \mu \mathrm{m}$ in the $z$ direction, and $12 d=4.44 \mu \mathrm{m}$ in the $y$ direction. To achieve a volume fraction of usually $\Phi=35 \%$, as in the experiment, we need to simulate 9241 spheres. Our shear direction is the $x$ direction, and the velocity gradient of the shear flow points in the $z$ direction; in other words, we shear the upper and lower $x y$ plane with respect to each other in the $x$ direction. We use periodic boundaries in the $x$ and $y$ directions and closed boundaries in the $z$ direction for both fluid and MD particles. The energy supplied by the shear force is dissipated by means of a Monte Carlo thermostat described in Refs. $[30,87]$. It acts on the fluid particles as well as on the MD particles and conserves the momentum in each SRD cell.

\section{EXPERIMENTAL SETUP}

Experiments are carried out with high-purity (99.97\%) $\alpha-\mathrm{Al}_{2} \mathrm{O}_{3}$ powder (RCHP DBM, Baikowski Malakoff Industries, Inc., USA). The mean particle diameter is $0.367 \mu \mathrm{m}$ (Coulter LS Particle Size Analyzer) and the size distribution is narrow $\left(d_{10}=0.176 \mu \mathrm{m}, d_{90}=0.664 \mu \mathrm{m}\right)$. The powder is suspended in bidistilled water (Merck, Germany). The suspension is then dispersed with alumina balls in a ceramic container for $24 \mathrm{~h}$ at a low rotational speed to keep the abrasion low. Subsequently, the suspension is degassed at 50 mbar under agitation. Then, in order to reduce the ionic strength to the desired degree, the suspension is purified by the dialysis technique. In this way, the majority of ions are removed and a background electrolyte of a very low salt concentration $\left(5 \times 10^{-4} \mathrm{~mol} / \mathrm{l}\right)$ is obtained for suspensions of high solids loading. Starting from this master suspension, suspensions with increased ionic strength are obtained by adding different amounts of dry ammonium chloride $\mathrm{NH}_{4} \mathrm{Cl}$ (Merck, Germany). The $p \mathrm{H}$ of the suspensions is adjusted to $p \mathrm{H}=6$ with 0.1 and $1 \mathrm{~mol} / 1$ hydrochloric acid $\mathrm{HCl}$ (Merck, Germany), if necessary. Thereby, the ionic strength and $p \mathrm{H}$ are revised by use of a laboratory $p \mathrm{H}$ and conductivity meter (inoLab pH/Cond Level 2, WTW GmbH, Germany). The electrophoretic mobility of dilute suspensions is measured with a Coulter Delsa 440 SX. Irreversible aggregation due to inhomogeneous salt concentration is not of importance here. If the ionic strength is strongly increased and after that a second dialysis step is performed to remove the ions again, the original viscosity is restored.

The ion concentrations of selected ions are measured before and after dialysis using inductively coupled plasmaoptical emission spectroscopy (ICP-OES, Model JY 70 plus, France). The suspensions are characterized using a Viscolab LC10 rheometer (Viscolab LM rheometer with control unit Viscolab LC10, Physica, Germany) with a cup and bob or a double gap geometry. The measurements are either performed immediately after suspension preparation or they are stored on a roller bank to avoid sedimentation. Sedimentation during the experiment can be excluded, since it takes much longer than the whole experiment, and the shear forces are much larger than gravity. Shear rate controlled experiments are performed at a constant temperature of $20^{\circ} \mathrm{C}$. The 


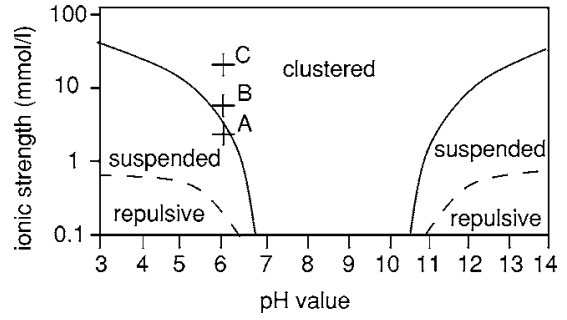

FIG. 1. Schematic stability diagram for volume fraction $\Phi$ $=35 \%$ in terms of $p \mathrm{H}$-value and ionic strength involving three different microstructures: A clustering regime due to van der Waals attraction, stable suspensions where the charge of the colloidal particles prevents clustering, and a repulsive structure for further increased electrostatic repulsion. This work concentrates on state $A$ $(p \mathrm{H}=6, I=3 \mathrm{mmol} / \mathrm{l})$ in the suspended region, state $B(p \mathrm{H}=6, I$ $=7 \mathrm{mmol} / \mathrm{l}$ ) close to the border but already in the clustered region, and state $C(p \mathrm{H}=6, I=25 \mathrm{mmol} / \mathrm{l})$ in the clustered region. The borders are not sharp transitions, but notable in a change of the shear viscosity.

suspensions are sheared at a constant shear rate of $\dot{\gamma}$ $=300 / \mathrm{s}(\mathrm{Pe}=8.8)$ before starting the actual ramp measurement. In the experiments, the shear rate is increased up to $\dot{\gamma}=4000 / \mathrm{s} \quad(\mathrm{Pe}=117)$ and decreased again to zero. In this paper, whenever referring to a shear rate we also specify the Péclet number

$$
\mathrm{Pe}=6 \pi \eta R^{3} \dot{\gamma} / k_{B} T,
$$

to make it easier for the reader to compare our results to other data. When the suspensions are presheared, an occurring discrepancy between the measured viscosity in the increasing ramp and the decreasing one can be minimized. A detailed description of the experiments will be published elsewhere [17,61].

\section{RESULTS}

\section{A. Stability diagram}

Depending on the experimental conditions, one can obtain three different microstructures: a clustered region, a suspended region, and a repulsive structure. The charge regulation model allows us to quantitatively relate the interaction potentials to certain experimental conditions. A schematic picture of the stability diagram is shown in Fig. 1. Close to the isoelectric point $(p \mathrm{H}=8.7)$, the particles form clusters for all ionic strengths since they are not charged. At lower or higher $p \mathrm{H}$ values, one can prepare a stable suspension for low ionic strengths because of the charge, which is carried by the colloidal particles. At even more extreme $p \mathrm{H}$ values, one can obtain a repulsive structure due to very strong electrostatic potentials (up to $\zeta=170 \mathrm{mV}$ for $p \mathrm{H}=4$ and $I$ $=1 \mathrm{mmol} / 1$, according to our model). The repulsive structure is characterized by an increased shear viscosity. In the following, we focus on three states: state $A(p \mathrm{H}=6, I$ $=3 \mathrm{mmol} / \mathrm{l})$ is in the suspended region, state $B(p \mathrm{H}=6, I$ $=7 \mathrm{mmol} / \mathrm{l})$ is a point already in the clustered region but still close to the border, and state $C(p \mathrm{H}=6, I=25 \mathrm{mmol} / \mathrm{l})$ is located well in the clustered region.
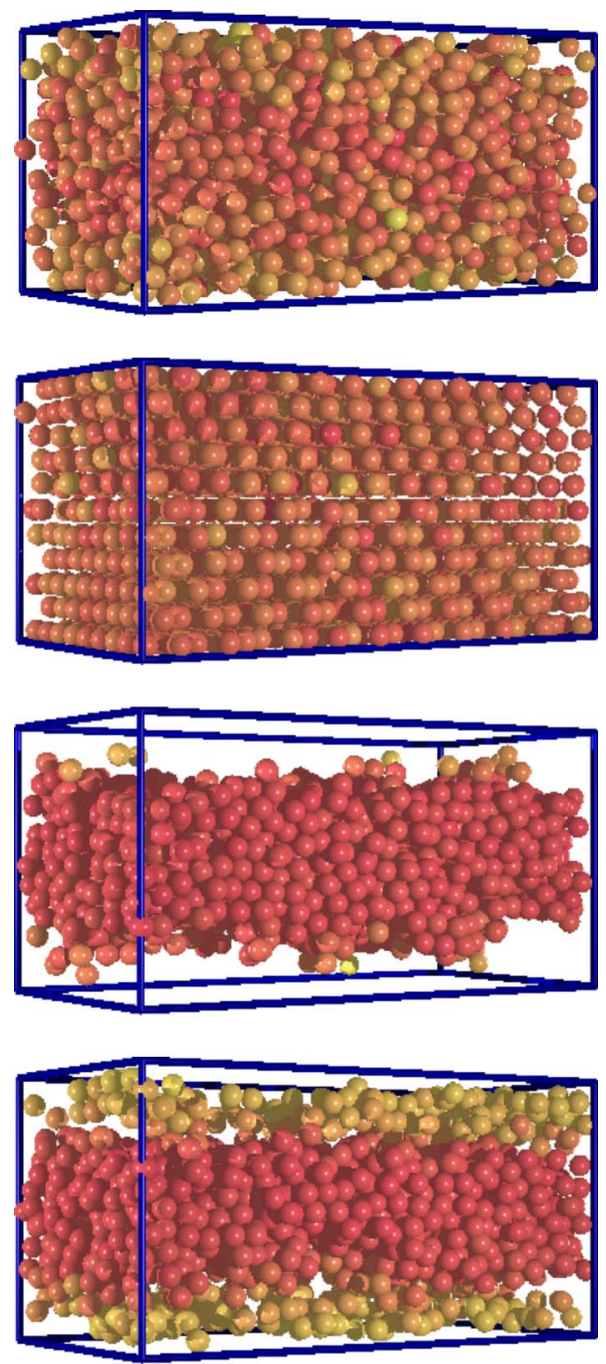

FIG. 2. (Color online) Images of four different cases. For better visibility we have chosen smaller systems than we usually use for the calculation of the viscosity. The colors denote velocities: Dark particles are slow, bright ones move fast. The potentials do not correspond exactly to the cases $A-C$ in Fig. 1, but they show qualitatively the differences between the different states: (a) Suspension like in state $A$, at low shear rates. (b) Layer formation, which occurs in the suspension (state $A$ ) at high shear rates and in the repulsive regime already at moderate shear rates. (c) Strong clustering, like in state $C$, so that the single cluster in the simulation is formed. (d) Weak clustering close to the border like in state $B$, where the cluster can be broken into pieces, which follow the flow of the fluid (plug flow).

Some typical examples for the different microstructures are shown in Figs. 2(a)-2(d). These examples are meant to be illustrative only and do not correspond exactly to the cases $A-C$ in Fig. 1 denoted by uppercase letters. In the suspended case (a), the particles are mainly coupled by hydrodynamic interactions. One can find a linear velocity profile and a slight shear thinning. If one increases the shear rate $\dot{\gamma}>500 / \mathrm{s}(\mathrm{Pe}>15)$, the particles arrange in layers. The same can be observed if the Debye-screening length of the electrostatic potential is increased (b), which means that the solvent contains fewer ions $(I<0.3 \mathrm{mmol} / \mathrm{l})$ to screen the par- 
ticle charges. On the other hand, if one increases the salt concentration, electrostatic repulsion is screened even more, and attractive van der Waals interaction becomes dominant $(I>4 \mathrm{mmol} / \mathrm{l})$. Then the particles form clusters (c), and viscosity rises. A special case, called "plug flow," can be observed for high shear rates, where it is possible to tear the clusters apart, and smaller parts of them follow with the flow of the solvent (d). This happens in our simulations for $I$ $=25 \mathrm{mmol} / \mathrm{l}$ (state $C$ ) at a shear rate of $\dot{\gamma}>500 / \mathrm{s}$ $(\mathrm{Pe}>15)$. However, as long as there are only one or two big clusters in the system, it is too small to expect quantitative agreement with experiments. In these cases, we have to focus on state $B(I=7 \mathrm{mmol} / \mathrm{l})$ close to the border.

In our simulations, we restrict ourselves to the region around $p \mathrm{H}=6$ where we find the border between the suspended region and the clustered regime at about $I$ $=4 \mathrm{mmol} / \mathrm{l}$ in the simulations as well as in the experiments. Also the shear rate dependence of the viscosity is comparable in simulations and experiments as discussed in Sec. VII C.

In Ref. [62], a qualitative stability diagram similar to Fig. 1 has been shown. The borders there are shifted, since they depend on the threshold value for which one defines that the viscosity has increased. Correspondingly, if one is less sensitive to the viscosity increase, one would still consider the system to be suspended, if only weak cluster formation takes place.

\section{B. Total energy}

In our simulations, we calculate the total energy, because it can be used as a tool to check if the response of the simulation to the variation of any parameter is consistent with the expectations, e.g., a decrease of the surface charge on the colloidal particles should cause the secondary minimum of the DLVO potential to become deeper and thus decrease the total energy, but if the total energy increases, this can be an indication for numerical instabilities.

The total energy comprises the kinetic energy of both fluid and colloidal particles, including thermal motion on the microscopic level, as well as the potential energy due to Coulomb repulsion, van der Waals attraction, and Hertz contact forces. Our simulations are carried out at room temperature $(T=295 \mathrm{~K})$ and constant volume fraction. Supposing a linear velocity profile, the kinetic energy increases quadratically with the shear rate $\dot{\gamma}$. This can be observed if the electrostatic repulsion is, on the one hand, strong enough to prevent cluster formation due to van der Waals attraction and, on the other hand, weak enough, so that the colloidal particles can move relatively freely without undergoing a glass transition or crystallization.

If the interactions are strongly repulsive, i.e., in the case of very low salt concentration, where the Debye-screening length is large, one can see an extra contribution of the electrostatic repulsion to the total energy. If the volume fraction is low, the particles can still find a configuration in which the mean nearest-neighbor distance is larger than the interaction range of the repulsion. But, if the volume fraction is increased, the particles have to be packed closer, which leads

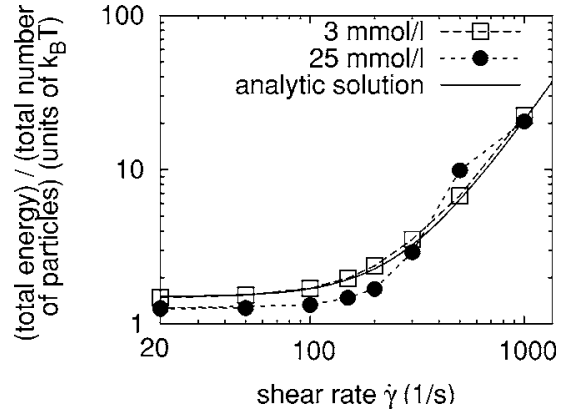

FIG. 3. Total energy depending on the shear rate $\dot{\gamma}$ for the states $A(I=3 \mathrm{mmol} / \mathrm{l})$ and $C(I=25 \mathrm{mmol} / \mathrm{l})$ of Fig. 1 . In state $A$ the system is a stable suspension; in state $C$ cluster formation reduces the total energy at low shear rates. At $\dot{\gamma}=500 / \mathrm{s}(\mathrm{Pe}=15)$, the cluster can be broken up into two parts moving in opposite directions. The two solid bodies have a larger kinetic energy than the suspension with a linear velocity profile. This explains the crossover of the two curves. For even higher $\dot{\gamma}$, the clusters are broken up in more pieces, leading ultimately to the same structure as for the suspended state $A$. The energy axis has been scaled by the total number of particles (fluid particles plus colloidal particles) and plotted in units of $k_{B} T$. The solid line is the analytical solution [Eq. (18)] for a linear velocity profile; the dashed lines are a guide to the eye.

to a constant positive offset to the total energy. It only depends on the potentials and on the volume fraction, but not on the shear rate.

In a similar way as for repulsive interactions, one can understand the negative energy contribution in the case of high salt concentrations: The DLVO potentials contain a minimum where attractive Van der Waals interaction is stronger than electrostatic repulsion. Then the particles form clusters and "try" to minimize their energy. In Fig. 3, for small shear rates the values for the energy in the clustered case of state $C(I=25 \mathrm{mmol} / \mathrm{l})$ are lower than for the suspended case of state $A(I=3 \mathrm{mmol} / \mathrm{l})$. We have plotted the total energy divided by the total number of all particles (fluid particles plus colloidal particles) in units of $k_{B} T$. For $\dot{\gamma} \rightarrow 0$, the energy per particle approaches $\frac{3}{2} k_{B} T$ as one would expect in 3D. The solid line in Fig. 3 is the sum of $\frac{3}{2} k_{B} T$ per particle with the kinetic energy of a fluid with a linear velocity profile,

$$
E_{\mathrm{tot}}=\frac{3}{2} k_{B} T N_{\mathrm{tot}}+\frac{1}{24} V \bar{\varrho} \dot{\gamma}^{2} L_{z}^{2},
$$

where $N_{\text {tot }}$ is the total number of both fluid and colloidal particles, $V$ denotes the volume of the simulated system, $\bar{\varrho}$ is the averaged mass density of the suspension, and $L_{z}$ is the extension in the $z$ direction (perpendicular to the shear plane). State $A$ coincides very well with this curve.

For state $C$, the behavior is shear-rate-dependent: In contrast to the repulsive case, clusters can be broken up. This happens at a shear rate $\dot{\gamma}=500 / \mathrm{s}(\mathrm{Pe}=15)$ (see Fig. 3) where one obtains two clusters moving in opposite directions. Since in this case the resistance of the system decreases, the velocity of the two clusters becomes larger. Since both clusters are moved as a whole, their energy becomes even larger than in the suspended case. If one further increases the shear rate, no 


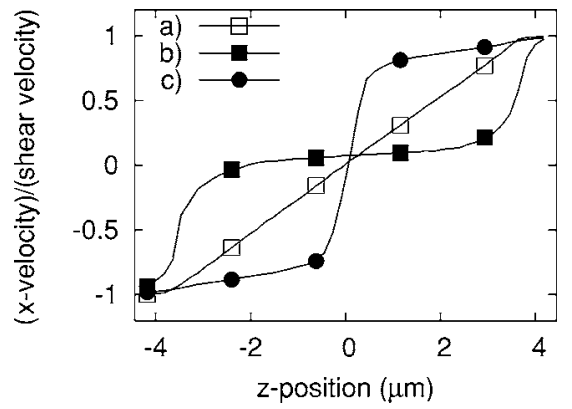

FIG. 4. Profiles of tangential velocity component $\left(v_{x}\right)$ in normal direction (z): (a) Linear profile in the suspended regime, state $A$ of Fig. $1(I=3 \mathrm{mmol} / \mathrm{l})$ at $\dot{\gamma}=500 / \mathrm{s}(\mathrm{Pe}=15)$. (b) Cluster formation in state $C(I=25 \mathrm{mmol} / \mathrm{l})$ at $\dot{\gamma}=100 / \mathrm{s}(\mathrm{Pe}=2.9)$. In principle, one could determine the viscosity of one single cluster from the central plateau, but this is not the viscosity found in experiments. There, one measures the viscosity of a paste consisting of many of these clusters. (c) Same as case (b) but with higher shear rate $(\dot{\gamma}=500 / \mathrm{s}$ $\mathrm{Pe}=15$ ). Hydrodynamic forces are large enough to break the cluster into two pieces. The velocity axis is scaled with the shear velocity $v_{S}$ for better comparability.

(big) clusters can form anymore, and the energies for both salt concentrations are nearly the same and correspond to the kinetic energy of a suspension with a nearly linear velocity profile. For $\dot{\gamma}=1000 / \mathrm{s}(\mathrm{Pe}=29)$, state $C$ coincides with the analytic curve.

\section{Shear profile and shear viscosity}

In each of the three regimes a typical velocity profile of the shear flow occurs. For the suspended microstructure, one finds a linear velocity profile [Fig. 4(a)] with nearly Newtonian flow. The particles are distributed homogeneously, thus the density profile is structureless [Fig. 5(a)]. The motion of the particles is only weakly coupled by the hydrodynamic forces. At high enough shear rates $[\dot{\gamma}>500 / \mathrm{s}(\mathrm{Pe}>15)]$, the particles arrange in layers parallel to the shear plane, as can also be seen in the density profile [Fig. 5(b)]. This arrangement minimizes collisions between the particles. As a result, the shear viscosity decreases, as shown in Fig. 6, which we discuss more in detail below. Shear-induced layer formation has been reported in the literature for different experiments [63-67] and Stokesian dynamics simulations $[19,68]$. For low shear rates, Brownian motion disturbs the layers or prevents their formation. As shown in Ref. [19], hydrodynamic forces can destroy them as well, if the shear rate is high enough. In our simulations, we do not reach these conditions. In the simulations, shear rates up to $2000 / \mathrm{s}(\mathrm{Pe}=59)$ can be realized before limitations of the simulation method influence the results. The increment of the shear angle in one SRD time step $\gamma=\dot{\gamma} \tau_{\mathrm{SRD}}$ amounts to about $\pi / 4$ then, i.e., the offset in the $x$ direction between two neighboring layers of SRD cells in the $z$ direction amounts to one cell per SRD time step.

Furthermore, the volume fraction and the interaction range of the electrostatic repulsion, or the ionic strength, respectively, influence the layer formation: In the repulsive regime, the layers are more clearly already at moderate shear
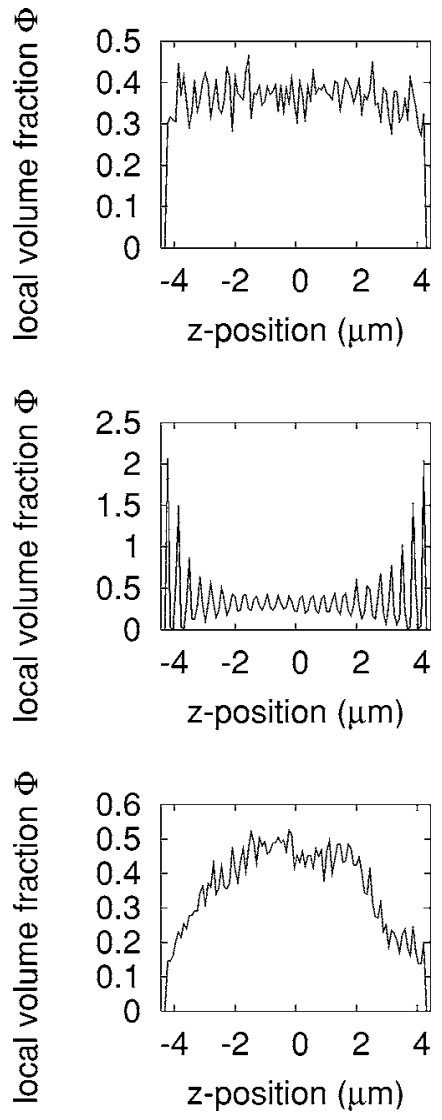

FIG. 5. Density profiles: (a) Suspended case: State $A$ in Fig. 1 $(I=3 \mathrm{mmol} / \mathrm{l})$, at low shear rates $[\dot{\gamma}=50 / \mathrm{s}(\mathrm{Pe}=1.5)]$. The density distribution is homogeneous. (b) Shear induced layer formation: This is state $A$ as in graph (a) of this figure, but for a high shear rate $[\dot{\gamma}=1000 / \mathrm{s} \quad(\mathrm{Pe}=29)]$. (c) Strong attractive forces in state $C(I=25 \mathrm{mmol} / \mathrm{l})$ : For low shear rates $[\dot{\gamma}=50 / \mathrm{s}(\mathrm{Pe}=1.5)]$ only one central cluster is formed, which is deformed slowly.

rates. It can be excluded that the effect is purely a finite-size effect, since for unsheared suspensions no layers can be observed, at least some particle diameters from the walls. In the repulsive regime, the particles try to optimize their local structure, but a long-range order as in the case of a sheared system cannot be seen.

In the clustered regime, the clusters move in the fluid as a whole. They are deformed, but since the interparticle forces are stronger than the hydrodynamic forces, the cluster moves more like a solid body than like a fluid. Often there is one big cluster that spans the whole system. The density profile [Fig. 5(c)] increases in the central region and decays at the regions close to the border, since particles from there join the central cluster. When averaging the velocity profile in the shear flow, one finds a very small velocity gradient in the center of the shear cell and fast-moving particles close to the wall, where the shear is imposed [Fig. 4(b)]. The velocity profile is nonlinear on the length scale of the simulations. In the experiment, the physical dimensions are much larger and therefore the velocity profile can become approximately linear again if the system consists of many large clusters. However, due to the computational effort in simulations it is impossible today to measure the shear viscosity for these 


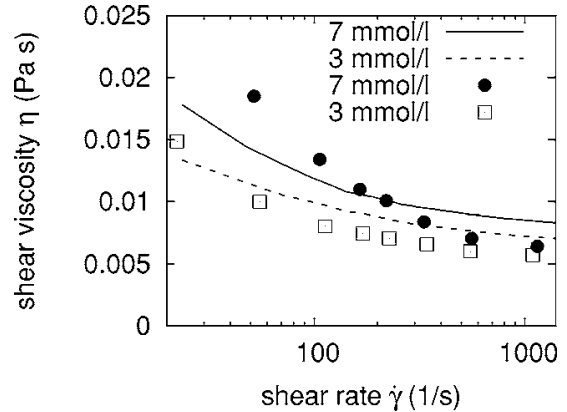

FIG. 6. Comparison between simulation and experiment: viscosity in dependence of the shear rate for the states $A(I=3 \mathrm{mmol} / \mathrm{l})$ and $B(I=7 \mathrm{mmol} / \mathrm{l})$ of Fig. 1 . Note: shear thinning is more pronounced for the slightly attractive interactions in state $B$ than for the suspended state $A$. Lines denote experimental data [17], points are results from our simulations.

strongly inhomogeneous systems. We have scaled our system by a factor of 2 in the $x$ and $z$ directions (keeping the volume fraction $\Phi=35 \%$ constant), but we still observe one big cluster after some hundreds of SRD time steps, i.e., finite-size effects are still present in our simulations.

Closer to the border, clusters can then be broken up into small pieces by the hydrodynamic forces at least for high shear rates. In state $C$ of Fig. 1, this happens for the first time at $\dot{\gamma}=500 / \mathrm{s}(\mathrm{Pe}=15)$, so that one can find two clusters in the system moving in opposite directions. The velocity profile of this case is shown in Fig. 4(c). For even higher shear rates or closer to the border (e.g., state $B$ ), the clusters are broken into smaller pieces. Then, they move in the shear flow with an approximately linear velocity profile. Due to van der Waals attraction, the system resists with stronger shear forces and the viscosity is higher than in the suspended case (Fig. 6).

In Fig. 6, the simulation results are shown together with the experimental results, both for the two cases of a slightly clustered system in state $B(I=7 \mathrm{mmol} / \mathrm{l})$ and a suspension (state $A, I=3 \mathrm{mmol} / \mathrm{l}$ ). For the suspension (state $A$ ), the viscosity decreases with the shear rate ("shear thinning"). The experimental data and the simulation are consistent within the accuracy of our model. There are several reasons why our model does not exactly fit the measurements: The most insecure factor that enters into the comparison is the measurement of the $\zeta$ potential. Starting from this point, we set up our charge regulation model to extrapolate to different salt concentrations, assuming two reactions the only processes that determine the surface charge of the colloidal particles. Furthermore, we have monodisperse spherical particles, which is another simplification in our model. Then, the lubrication force as a correction for the finite resolution of the fluid method can only recover to a certain degree the hydrodynamics on smaller length scales than the cell size of the fluid simulation, e.g., we have not implemented other modes of lubrication than the "squeezing mode" [Eq. (3)].

However, we have done several tests where we have simulated systems with size and charge polydispersity in the order of magnitude corresponding to the experimental conditions. We have tested different sorts of boundary condi-

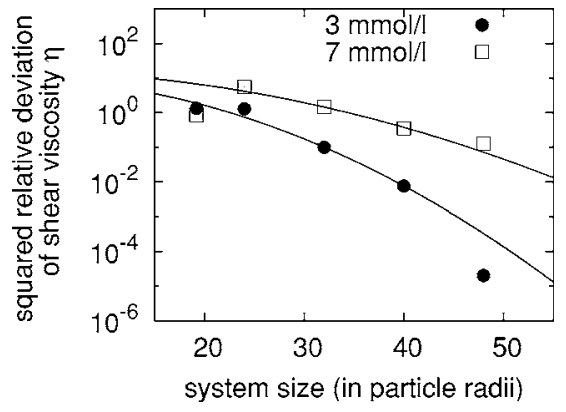

FIG. 7. Discrepancy between simulated viscosity and measurement for states $A$ and $B$ of Fig. 1 for different system sizes at low shear rates. The plot shows squared relative differences against $z$ extension of the simulation volume. The lines are a guide to the eye.

tions, different ways to implement shear, and different coupling methods between fluid and particles. In most of the tests, the achieved shear viscosity in the simulation did not change notably. In our experience, the way shear is imposed and the particle size have the largest influence on the result.

Finally, one has to keep in mind that the viscosity of the suspension can be varied by more than one order of magnitude, e.g., by changing the ionic strength. In this context, the deviations between simulation an experiment are small.

For the slightly clustered case (state $B$ ), an increase of the shear viscosity, compared to the suspended case, can be observed in the experiment as well as in the simulations. Shear thinning becomes more pronounced, because clusters are broken up, as mentioned above. However, the shear-rate dependence is stronger in the simulations than in the experiment. This can be the first indication of finite-size effects.

We have studied the dependence of the simulated shear viscosity in dependence of the system size. The effect is most important for low shear rates and thus we carried out several simulations for state $A$ at $\dot{\gamma}=20 / \mathrm{s}(\mathrm{Pe}=0.6)$ and for state $B$ at $\dot{\gamma}=50 / \mathrm{s} \quad(\mathrm{Pe}=1.4)$. We have chosen these values because clustering is already too strong in state $B$ at $\dot{\gamma}=20 / \mathrm{s}$ $(\mathrm{Pe}=0.6)$ to reasonably determine a viscosity, and the system size dependence becomes too small for $\dot{\gamma}=50 / \mathrm{s} \quad(\mathrm{Pe}=1.4)$ were state $A$. In Fig. 7, we plot the squared relative deviation between simulation and measurements against the system size. We do not know if the simulation results would exactly converge to the measured values if the simulated system is large enough. However, the figure shows the trend that the deviation becomes smaller for larger system sizes, but to reach in state $B$ the same accuracy as in state $A$ one would at least have to double the system size in each dimension. It then takes approximately twice as long for the system to relax to a steady state, resulting in a factor of 16 in the computational effort. This indicates that each single point of Fig. 6 would need approximately 3000 CPU hours. For smaller shear rates or even deeper in the clustered regime of the stability diagram, e.g., in state $C(I=25 \mathrm{mmol} / \mathrm{l})$, the finite-size effects become more pronounced-ending up in the extreme case of only one big cluster existing in the system. For simulations with good accuracy, the effort again increases at least by the same factor. 
Unfortunately, this would be the most interesting case with respect to soil mechanics and to understand landslides, which was our initial motivation. Anyhow, if we compare state $A$ and state $B$, shear thinning becomes stronger with increasing ionic strength. In the experiments, the effect becomes much stronger for larger ionic strengths (up to $I$ $=65 \mathrm{mmol} / \mathrm{l}$ ), where the viscosity for low shear rates is increased by more than a factor of 10 . However, the fact that there is shear thinning and that it depends on the ionic strength is an interesting result, if we recall the fact that the lubrication force in our simulation can be interpreted as a velocity-dependent damping force, which becomes stronger for higher relative velocities. Therefore, one would intuitively expect shear thickening.

Finally, the limitations of the DLVO theory have to be taken into account. DLVO potentials are derived for dilute suspensions and hence large particle distances. This is not fulfilled in our case - and even less inside the clusters. There are theoretical attempts that address the shortcomings of DLVO theory: Explicit simulation of micro-ions [69], density- functional theory [70-72], response theory [73-76], Poisson-Boltzmann cell models [77-79], and full PoissonBoltzmann theory [80-83], but they have other disadvantages - most of them require a large computational effort. For Poisson-Boltzmann cell models, one assumes homogeneously distributed colloidal particles, so that each of them can be regarded as a representative single particle in a Wigner-Seitz cell. Additionally, depending on the level Poisson-Boltzmann theory is included, a mysterious phase separation could be identified as an artifact of linearization [79]. Full Poisson-Boltzmann theory would require the calculation of the local potential, not only as done in our charge regulation model in the beginning of the simulation, but for the whole simulation box and in each time step. This would in principle provide a better description of the real system, but the computational effort would be much larger, making simulations of several thousands of particles impossible. The same applies to the approach of including the micro-ions explicitly in the simulation. One could obtain three- (many-) body interactions from full Poisson-Boltzmann theory and try to include them as a lookup table in the simulation. However, one would have to decrease the system size to keep the computational effort affordable. For our simulations we need relatively simple pair potentials to keep the computational costs within a limit. Nevertheless, the overall behavior can be reproduced by the simulation on a semiquantitative level. The reason for that might be the fact that in some of the above-mentioned theoretical attempts (density-functional theory and cell models), DLVO-like potentials are obtained with a renormalized charge and screening length. In our charge regulation model, we merely adjust a renormalized charge to the measurements of the $\zeta$ potential. This may be a general explanation why DLVO potentials can often be used, although the assumptions for DLVO theory are not fulfilled [52,54,84-86].

We have carried out simulations in the repulsive region of the stability diagram as well. We find layers parallel to the shear plane in analogy to Fig. 5(b). In contrast to the suspended regime, in the repulsive regime the layer structure is present-at least locally, but orientationally disordered-

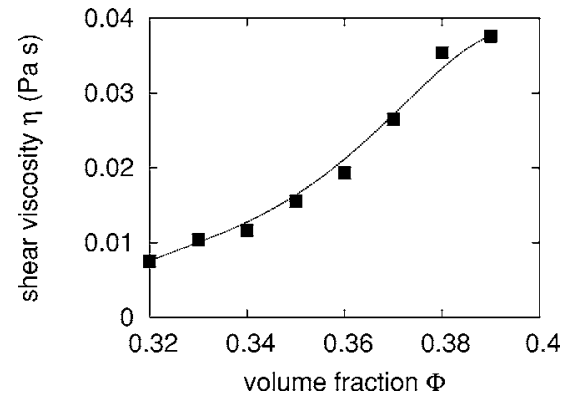

FIG. 8. Viscosity vs volume fraction for the repulsive region $(p \mathrm{H}=6$ and $I=0.3 \mathrm{mmol} / \mathrm{l})$. The shear rate was $\dot{\gamma}=100 / \mathrm{s}$ $(\mathrm{Pe}=2.9)$. The points are simulation results, the line is a guide to the eye.

even if no shear is applied. If shear flow is present, the shear plane marks one orientation that the layer structure adopts. In some cases, for very low ionic strengths one can observe shear bands so that the velocity gradient and thus the viscosity vary strongly in the system. Again, in the experiment, physical dimensions are much larger and on that length scale the velocity profile might be assumed to be linear when enough shear bands are in the system. The shear force and hence the viscosity increase with respect to the suspended regime, due to electrostatic repulsion. One can consider the particles together with the interaction range as soft spheres with an effective radius of the interaction range of the electrostatic repulsion. This effective radius in our case can be about $25 \%$ larger than the particle radius. Therefore, a transition to a repulsive structure already occurs in our systems between $35 \%$ and $40 \%$ volume fraction. Because of the smooth shape of the exponentially screened Coulomb potential, it is not a sharp glass transition as for hard spheres, but smooth and shear-rate-dependent as well. In Fig. 8, we have shown the dependence of the viscosity on the volume fraction for $p H=6$ and $I=0.3 \mathrm{mmol} / 1$. Starting at $\Phi=0.3$, the shear viscosity starts to increase and reaches a value one decade larger beyond $\Phi=0.4$.

\section{SUMMARY AND OUTLOOK}

We have shown how to relate DLVO potentials to the conditions in a real aqueous suspension of $\mathrm{Al}_{2} \mathrm{O}_{3}$ particles. The behavior of shear viscosity has been studied in experiments and in simulations. We have found shear thinning due to a layer formation on the microscopic scale in the case of a suspension.

If a clustered system is sheared, clusters are broken up into pieces by the imposed shear, which leads to a stronger shear thinning than in the suspended case. Close to the border, we are able to reproduce the measured shear viscosity in the simulation.

Deep in the clustered regime, we have found that our particles form one big cluster in the system that can be broken up by the hydrodynamic forces of the shear flow. For strongly clustered systems at low shear rate, which would be the most interesting case for soil mechanics, there are strong finite-size effects. One attempt to address this problem is to 
increase the size of the simulated system. As we have shown with our work, the computational effort increases to an extend that a parallelized simulation code would be necessary. However, this might not be sufficient, since in both cases, for low shear rates and for strongly attractive interactions, the finite-size effects become stronger. Since in these simulations a considerable amount of the computing time is consumed by the particles inside a cluster, one could think of a more coarse-grained description of the clusters. Nevertheless, input data for such a model could be obtained using our present simulation code. Depending on the model, one might need information about the shear resistance of a single cluster, depending on the cluster size and shape. This would be very difficult to measure, but it could be calculated in a small simulation using our combined MD and SRD algorithm.

\section{ACKNOWLEDGMENTS}

This work has been financed by the German Research Foundation (DFG) within the project DFG-FOR 371 "Peloide." We thank G. Gudehus, G. Huber, M. Külzer, L. Harnau, and S. Richter for valuable collaboration. Parts of this work have resulted from a collaboration with the group of A. Coniglio, Naples, Italy. M. H. thanks him and his group for their hospitality and for the valuable support during his stay there. M. H. thanks the DAAD for the financial support that enabled him to stay. H. J. H. thanks the Alexander von Humbolt Stiftung and the Max Planck Gesellschaft for the Max Planck prize. M. B. is deeply indebted to M. Biesheuvel and S.-H. Behrens for helpful discussions on charge regulation models. The computations were performed on the IBM p690 cluster at the Forschungszentrum Jülich, Germany.
[1] H. van Olphen, An Introduction to Clay Colloid Chemistry, 2nd ed. (Krieger Publishing Company, Malabar, FL, 1991).

[2] R. Agra, E. Triac, and L. Boequet, Eur. Phys. J. E 15, 345(2004).

[3] F. M. van der Kooij, K. Kassapidou, and H. N. W. Lekkerkerker, Nature (London) 406, 868 (2000).

[4] M. Dijkstra, J. P. Hansen, and P. A. Madden, Phys. Rev. Lett. 75, 2236 (1995).

[5] M. Dijkstra, J.-P. Hansen, and P. A.Madden, Phys. Rev. E 55, 3044 (1997).

[6] E. DiMasi, J. O. Fossum, T. Gog, and C. Venkataraman, Phys. Rev. E 64, 061704 (2001).

[7] J. Mahanty and B. W. Ninham, Dispersion Forces (Academic Press, London, 1996).

[8] G. Lagaly, O. Schulz, and R. Zimehl, Dispersionen und Emulsionen (Dr. Dietrich Steinkopff Verlag, Darmstadt, Germany, 1997).

[9] D. J. Shaw, Introduction to Colloid and Surface Chemistry (Butterworth-Heinemann Ltd., Oxford, 1992).

[10] I. D. Morrison and S. Ross, Colloidal Dispersions: Suspensions, Emulsions and Foams (John Wiley and Sons, New York, 2002).

[11] R. J. Hunter, Foundations of Colloid Science (Oxford University Press, Oxford, UK 2001).

[12] V. Trappe, V. Prasad, L. Cipelletti, P. N. Segre, and D. A. Weitz, Nature (London) 411, 772 (2001).

[13] N. Sator, E. del Gado, and A. Coniglio, Physica A 358, 239 (2005).

[14] I. Saika-Voivod, E. Zaccarelli, F. Sciortino, S. V. Buldyrev, and P. Tartaglia, Phys. Rev. E 70, 041401 (2004).

[15] A. M. Puertas, M. Fuchs, and M. E. Cates, J. Chem. Phys. 121, 2813 (2004).

[16] F. Mallamace, S. H. Chen, A. Coniglio, L. de Arcangelis, E. Del Gado, and A. Fierro, Phys. Rev. E 73, 020402(R) (2006).

[17] J. Reinshagen, R. C. D. Cruz, R. Oberacker, and J. Hoffmann (unpublished).

[18] J. F. Brady and G. Bossis, Annu. Rev. Fluid Mech. 20, 111 (1988)

[19] T. N. Phung, J. F. Brady, and G. Bossis, J. Fluid Mech. 313,
$181(1996)$

[20] J. F. Brady, J. Chem. Phys. 99, 567 (1993).

[21] A. Sierou and J. F. Brady, J. Fluid Mech. 448, 115 (2001).

[22] A. Sierou and J. F. Brady, J. Fluid Mech. 506, 285 (2004).

[23] L. E. Silbert, J. R. Melrose, and R. C. Ball, Phys. Rev. E 56, 7067 (1997).

[24] M. Hütter, J. Colloid Interface Sci. 231, 337 (2000).

[25] M. Hütter, Ph.D. thesis, Swiss Federal Institute of Technology, Zurich (1999).

[26] A. J. C. Ladd and R. Verberg, J. Stat. Phys. 104, 1191 (2001).

[27] A. J. C. Ladd, J. Fluid Mech. 271, 285 (1994).

[28] A. J. C. Ladd, J. Fluid Mech. 271, 311 (1994).

[29] A. Komnik, J. Harting, and H. J. Herrmann, J. Stat. Mech.: Theory Exp. 2004, 12003 (2004).

[30] M. Hecht, J. Harting, T. Ihle, and H. J. Herrmann, Phys. Rev. E 72, 011408 (2005).

[31] Y. Inoue, Y. Chen, and H. Ohashi, J. Stat. Phys. 107, 85 (2002).

[32] J. T. Padding and A. A. Louis, Phys. Rev. Lett. 93, 220601 (2004).

[33] E. J. W. Vervey and J. T. G. Overbeek, Theory of the Stability of Lyophobic Colloids (Elsevier, Amsterdam, 1948).

[34] B. V. Derjaguin and L. D. Landau, Acta Physicochim. URSS 14, 633 (1941).

[35] W. B. Russel, D. A. Saville, and W. Schowalter, Colloidal Dispersions (Cambridge Univ. Press, Cambridge, 1995).

[36] J. A. Lewis, J. Am. Ceram. Soc. 83, 2341 (2000).

[37] L. Bocquet, E. Trizac, and M. Aubouy, J. Chem. Phys. 117, 8138 (2002).

[38] A. Malevanets and R. Kapral, J. Chem. Phys. 110, 8605 (1999).

[39] A. Malevanets and R. Kapral, J. Chem. Phys. 112, 7260 (2000).

[40] M. Ripoll, K. Mussawisade, R. G. Winkler, and G. Gompper, Europhys. Lett. 68, 106 (2004).

[41] I. Ali, D. Marenduzzo, and J. M. Yeomans, J. Chem. Phys. 121, 8635 (2004).

[42] H. Noguchi and G. Gompper, Phys. Rev. Lett. 93, 258102 (2004). 
[43] K. Tucci and R. Kapral, J. Chem. Phys. 120, 8262 (2004).

[44] A. Lamura, G. Gompper, T. Ihle, and D. M. Kroll, Europhys. Lett. 56, 319 (2001).

[45] E. Tuzel, M. Strauss, T. Ihle, and D. M. Kroll, Phys. Rev. E 68, 036701 (2003).

[46] E. Falck, J. M. Lahtinen, I. Vattulainen, and T. Ala-Nissila, Eur. Phys. J. E 13, 267 (2004).

[47] R. G. Winkler, K. Mussawisade, M. Ripoll, and G. Gompper, J. Phys.: Condens. Matter 16, S3941 (2004).

[48] J. T. Padding and A. A. Louis, e-print cond-mat/0603391.

[49] R. Yamamoto, K. Kim, Y. Nakayama, K. Miyazaki, and D. R. Reichman, e-print cond-mat/0604404.

[50] P. Debye and E. Hückel, Phys. Z. 24, 185 (1923).

[51] D. A. McQuarrie, Statistical Mechanics (Univ. Science Books, Sausalito, CA, 2000).

[52] S. Alexander, P. M. Chaikin, P. Grant, G. J. Morales, P. Pincus, and D. Hone, J. Chem. Phys. 80, 5776 (1984).

[53] M. Aubouy, E. Trizac, and L. Bocquet, J. Phys. A 36, 5835 (2003).

[54] E. Trizac, L. Bocquet, M. Aubouy, and H. H. von Grünberg, Langmuir 19, 4027 (2003).

[55] D. Chan, J. W. Perram, L. R. White, and T. W. Healy, J. Chem. Soc., Faraday Trans. 1 78, 1046 (1975).

[56] R. C. D. Cruz, Ph.D. thesis, University of Karlsruhe, Germany (2006).

[57] M. Evers, N. Garbow, D. Hessinger, and T. Palberg, Phys. Rev. E 57, 6774 (1998).

[58] P. Wette, H. J. Schöpe, and T. Palberg, J. Chem. Phys. 116, 10981 (2002).

[59] V. Lobaskin, B. Dünweg, and C. Holm, J. Phys.: Condens. Matter 16, S0953 (2004).

[60] V. Lobaskin, M. Medebach, T. Palberg, B. Duenweg, and C. Holm, e-print cond-mat/0601588.

[61] J. Reinshagen, Ph.D. thesis, University of Karlsruhe, Germany (2006).

[62] T. J. Graule, F. H. Baader, and L. J. Gauckler, CFI, Ceram. Forum Int. 71, 314 (1994).

[63] B. J. Ackerson, J. B. Hayler, N. A. Clark, and L. Cotter, J. Chem. Phys. 84, 2344 (1986).

[64] B. J. Ackerson and P. N. Pusey, Phys. Rev. Lett. 61, 1033 (1988).
[65] B. J. Ackerson, C. G. DeKruif, N. J. Wagner, and W. B. Russel, J. Chem. Phys. 90, 3250 (1989).

[66] I. Cohen, T. G. Mason, and D. A. Weitz, Phys. Rev. Lett. 93, 046001 (2004).

[67] J. Vermant and M. J. Solomon, J. Phys.: Condens. Matter 17, R187 (2005).

[68] D. R. Foss and J. F. Brady, J. Fluid Mech. 407, 167 (2000).

[69] P. Linse, J. Chem. Phys. 113, 4359 (2000).

[70] H. Graf and H. Löwen, Phys. Rev. E 57, 5744 (1998).

[71] R. van Roij and J.-P. Hansen, Phys. Rev. Lett. 79, 3082 (1997).

[72] R. van Roij, M. Dijkstra, and J.-P. Hansen, Phys. Rev. E 59, 2010 (1999).

[73] M. J. Grimson and M. Silbert, Macromol. Phys. 74, 397 (1991).

[74] A. R. Denton, J. Phys.: Condens. Matter 11, 10061 (1999).

[75] A. R. Denton, Phys. Rev. E 62, 3855 (2000).

[76] A. R. Denton, Phys. Rev. E 70, 031404 (2004).

[77] H. H. von Grünberg, R. van Roij, and G. Klein, Europhys. Lett. 55, 580 (2001).

[78] M. Deserno and H. H. von Grünberg, Phys. Rev. E 66, 011401 (2002).

[79] M. N. Tamashiro and H. Schiessel, J. Chem. Phys. 119, 1855 (2003).

[80] R. W. Bowen and A. O. Sharif, Nature (London) 393, 663 (1998).

[81] J. J. Gray, B. Chiang, and R. Bonnecaze, Nature (London) 402, 750 (1999).

[82] J. Dobnikar, Y. Chen, R. Rzehak, and H. H. von Grünberg, J. Phys.: Condens. Matter 15, S263 (2003).

[83] J. Dobnikar, R. Rzehak, and H. H. von Grünberg, Europhys. Lett. 61, 697 (2003).

[84] T. Palberg, W. Mönch, F. Bitzer, R. Piazza, and T. Bellini, Phys. Rev. Lett. 74, 4555 (1995).

[85] R. Kjellander and D. J. Michell, Chem. Phys. Lett. 74, 76 (1992).

[86] E. Trizac, L. Bocquet, and M. Aubouy, Phys. Rev. Lett. 89, 248301 (2002)

[87] Note: The 2 in the denominator of Eq. (33) in Ref. [30] has to be a 3 for the $3 \mathrm{D}$ case. 